\title{
HyperNaut: a navigator for the hyperbolic plane
}

\section{Benjamin Wilson $^{1}$}

1 Lateral $\mathrm{GmbH}$

DOI: $10.21105 /$ joss. 01240

\section{Software}

- Review ¿

- Repository ¿

- Archive c

Submitted: 01 February 2019

Published: 20 February 2019

\section{License}

Authors of papers retain copyright and release the work under a Creative Commons Attribution 4.0 International License (CC-BY).

\section{Summary}

Several recent works have presented methods for embeddings graphs (Ben Chamberlain \& Clough, 2017) (Nickel \& Kiela, 2017) (Nickel \& Kiela, 2018) and words (Leimeister \& Wilson, 2018) (Dhingra, Shallue, Norouzi, Dai, \& Dahl, 2018) in hyperbolic space. HyperNaut provides researchers in machine learning with a much-needed mechanism for inspecting the hyperbolic embeddings resulting from their algorithms using a navigable visualisation on the Poincaré disc. Consisting entirely of client-side JavaScript, the software is able to run locally in any modern web browser.

The Poincaré disc displays the entirety of the hyperbolic plane within the familiar Euclidean unit disc. In practice, however, due to the finite resolution of both the computer display and the eye, points that are far from the focal point of the disc are no longer visible. Such point configurations occur frequently in hyperbolic embeddings, however. HyperNaut is built to address this problem. It provides a navigation mechanism for the hyperbolic plane, allowing the user to visit and explore far-off constellations of points. This is achieved via simple dragging motions on the Poincaré disc. These are able to effect arbitrary rotations and translations of the entire point configuration.

In addition to this primary use case, HyperNaut is a useful tool for gaining an understanding of hyperbolic space and of the Poincaré disc model, in particular. To this end, a point-dragging functionality is included. This permits the formation of arbitrary polygons, with edges being represented by geodesic arcs. Viewing such polygons from various perspectives (by applying translations and rotations) provides an understanding of the deformations inherent in the Poincaré disc model.

\section{Computational geometry on the hyperbolic plane}

There are various models of the hyperbolic plane. While the Poincaré disc model is used for display, the geometric computations of the software are carried out using the hyperboloid model. The exception to this is the calculation of a geodesic line segment, which is acheived using circle inversion through the boundary of the Poincare disc.

The point configuration can be manipulated either by dragging individual points or by dragging all points simultaneously. The computations entailed involve the exponential, logarithm and geodesic parallel-transport maps on the hyperboloid. The formulae for these maps are well-known, and can be found e.g. in (Leimeister \& Wilson, 2018). Equipped with these maps, the user interaction and the consequent computations are as follows:

Dragging an individual point: The point is selected by holding down the mouse button, but is not moved until the mouse button is released. While the mouse button 
is held down, the position to which the point would be mapped is indicated, along with the geodesic line segment connecting the current and updated versions of the point. The position of the mouse during drag specifies the tangent to the geodesic line along which it would move. The new position of the point is then the exponential of (the conformal scaling of) this tangent, computed at its original position.

Dragging all points: All points can be dragged simultaneously by dragging the background of the Poincaré disc. The positions of all points are continuously updated while the mouse button is held down. If $\mathrm{X}$ was the mouse position last round, and $\mathrm{Y}$ is the current mouse position, then the update is calculated as follows:

- the logarithm of each point of the configuration is computed at $\mathrm{X}$, yielding tangent vectors at $\mathrm{X}$;

- these are then parallel-transported along the geodesic connecting $\mathrm{X}$ to $\mathrm{Y}$, yielding tangent vectors at $\mathrm{Y}$;

- the exponential map at $\mathrm{Y}$ is applied to these tangent vectors, mapping the points of the configuration to their updated positions.

\section{References}

Ben Chamberlain, M. D., \& Clough, J. (2017). Neural embeddings of graphs in hyperbolic space. In Proceedings of the 13th international workshop on mining and learning with graphs (mlg). Retrieved from http://www.mlgworkshop.org/2017/paper/ MLG2017_paper_6.pdf

Dhingra, B., Shallue, C., Norouzi, M., Dai, A., \& Dahl, G. (2018). Embedding text in hyperbolic spaces. In Proceedings of the twelfth workshop on graph-based methods for natural language processing (textgraphs-12) (pp. 59-69). New Orleans, Louisiana, USA: Association for Computational Linguistics. doi:https://doi.org/10.18653/v1/w18-1708

Leimeister, M., \& Wilson, B. J. (2018). Skip-gram word embeddings in hyperbolic space. CoRR, abs/1809.01498. Retrieved from http://arxiv.org/abs/1809.01498

Nickel, M., \& Kiela, D. (2017). Poincaré embeddings for learning hierarchical representations. In I. Guyon, U. V. Luxburg, S. Bengio, H. Wallach, R. Fergus, S. Vishwanathan, \& R. Garnett (Eds.), Advances in neural information processing systems 30 (pp. 6338-6347). Curran Associates, Inc. Retrieved from http://papers.nips.cc/paper/ 7213-poincare-embed dings-for-learning-hierarchical-representations.pdf

Nickel, M., \& Kiela, D. (2018). Learning continuous hierarchies in the Lorentz model of hyperbolic geometry. In J. Dy \& A. Krause (Eds.), Proceedings of the 35th international conference on machine learning, Proceedings of machine learning research (Vol. 80, pp. 3779-3788). Stockholmsmässan, Stockholm Sweden: PMLR. Retrieved from http://proceedings.mlr.press/v80/nickel18a.html 